\title{
Staged Demodulation and Decoding
}

\author{
Luca Barletta, Maurizio Magarini, Arnaldo Spalvieri \\ Dipartimento di Elettronica e Informazione \\ Politecnico di Milano \\ Milan, Italy \\ Email: \{barletta,magarini,spalvier\}@elet.polimi.it
}

\begin{abstract}
Coding for the phase noise channel is investigated in the paper. Specifically, Wiener's phase noise, which induces memory in the channel, is considered. A general coding principle for channels with memory is that of interleaving two or more codes. The interleaved codes are decoded in sequence, using past decisions to help future decoding. The paper proposes a method based on this principle, and shows its benefits through numerical results obtained by computer simulation. Analysis of the channel capacity given by the proposed method is also worked out in the paper.
\end{abstract}

\section{INTRODUCTION}

Coherent demodulation of advanced coded modulation formats is a challenging task in classical radio transmission systems and in coherent optical transmission systems of new generation. Besides the common Additive White Gaussian Noise (AWGN), the performance of coherent demodulation can be strongly impaired by multiplicative phase noise. In the context of radio transmission, phase noise is induced by local oscillators used for up-conversion and down-conversion, and its impact on system performance is widely recognized from a long time. In the context of optical transmission, it is known that laser's phase noise is a Wiener process [1], and the Wiener model has been recently proposed in [2] also for the phase noise accumulated during nonlinear propagation, at least for the cases studied in that paper. Recent papers [3], [4], [5] address the problem of coherent demodulation in the presence of Wiener phase noise. Also, Wiener phase noise is adopted in [6], [7], [8] to assess the performance of iterative demodulation and decoding, while the capacity of the channel affected by Wiener phase noise is derived in [9]. Often, one is lead to introduce pilot symbols to aid carrier recovery in the presence of strong phase noise [6], [10], [11], the capacity of Wiener's phase noise channel with pilot symbols being studied in [12]. However, pilot symbols sacrifice spectral efficiency. As an alternative to pilot symbols, one can resort to differential demodulation methods as those proposed in [7], [13]. The trellisbased method of [13] in conjunction with iterative differential demodulation and decoding offers excellent performance at the expense of large complexity of signal processing, while the method based on Tikhonov parametrization [7] is less demanding, still offering good performance.

A general coding principle for channels with memory, as Wiener's phase noise channel is, is that of interleaving two or more codes. The interleaved codes are decoded in sequence by the so-called staged decoding technique, which uses past

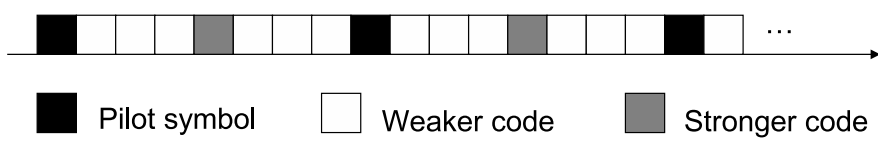

Fig. 1. Example of two-stage coded sequence with pilots according to equation (1).

decisions to help future decoding. The use of interleaving and staged decoding aided by past decisions has been proposed in [14] and expanded in [15] for the InterSymbol Interference (ISI) AWGN channel, where the generic stage consists of equalization and decoding. The general principle has then found application to a variety of channels with memory [16], [17].

The scheme proposed here relies upon interleaving of pilot symbols and coded symbols from two (or more) codes. Channel symbols of the more powerful code are demodulated and decoded first, then decisions on first-level coded symbols are used as pilot symbols in the successive demodulation and decoding stage. For instance, with two component block codes one can transmit through the channel the sequence

$$
\begin{aligned}
& \left(p, c_{2,1}, c_{2,2}, c_{2,3}, c_{1,1}, c_{2,4}, c_{2,5}, c_{2,6}\right) ; \\
& \left(p, c_{2,7}, c_{2,8}, c_{2,9}, c_{1,2}, c_{2,10}, c_{2,11}, c_{2,12}\right) ; \\
& (p, \cdots) ; \\
& \cdots \\
& \left(p, c_{2,6 N-5}, c_{2,6 N-4}, c_{2,6 N-3}, c_{1, N}, c_{2,6 N-2}, c_{2,6 N-1}, c_{2,6 N}\right) .
\end{aligned}
$$

In the above sequence, represented in Fig. 1, $p$ represents one pilot symbol and $c_{i, j}$ is the $j$-th symbol of the $i$-th level code $\mathcal{C}_{i}$. In (1), one frame is inserted between parentheses, the entire sequence consists of $N$ frames, the length of code $\mathcal{C}_{2}$ is $6 N$ while the length of code $\mathcal{C}_{1}$ is $N$. For the sake of concreteness, in the following only two-level constructions will be considered, the extension to multilevel constructions being straightforward.

The main novelty of our proposal is the application of the principle of interleaving and staged decoding to the phase noise channel, where the individual stage consists of demodulation and decoding. Compared to the previous literature on interleaving and staged decoding, specifically references [14], [15], [16], [17], other minor novelties that we can claim are the following. 
- While in [14] and [16] only one code is considered, here the use of a set of different channel codes is proposed.

- While in [14] the pattern of pilot symbols is a block of pilots with block length equal to the memory of the channel, here blocks of size much smaller than channel's memory are used. Pilot symbols are not considered in [15] and in [16].

- In the interleaving scheme presented here, a different number of symbols from different codes are interleaved in each frame, while in [15] and [17] the same number of symbols from different codes is interleaved in each frame.

The paper is organized as follows. Section II is devoted to the channel and system model. Section III reports the analysis of channel capacity. In Section IV the results that are obtained with the proposed method in contrast with adversary methods are shown. Finally, in Section V conclusions are drawn.

\section{Channel And System Model}

The $k$-th received sample $y_{k}$ is

$$
y_{k}=\left(x_{k}+w_{k}\right) e^{j \theta_{k}},
$$

where $x_{k}$ is the $k$-th transmitted symbol, $w_{k}$ is the $k$-th sample of Additive White Gaussian Noise (AWGN), and $\theta_{k}$ is the $k$-th sample of phase noise. The phase noise is hereafter modeled as a discrete-time Wiener process

$$
\theta_{k}=\left[\theta_{k-1}+\gamma v_{k}\right] \bmod 2 \pi, \quad k=1,2, \cdots,
$$

where $\gamma>0$ is a known parameter, $\theta_{0}$ is uniformly distributed in $[0,2 \pi)$, and $v_{k}$ is the $k$-th sample of white Gaussian noise with zero mean and unit variance. The phase evolution given in (2) occurs when the power spectral density of the continuoustime complex exponential $e^{j \theta(t)}$, whose samples at symbol frequency generate the sequence $e^{j \theta_{k}}$, is the Lorentzian function

$$
\mathcal{L}(f)=\frac{4 \gamma^{2} T}{\gamma^{4}+16 \pi^{2} f^{2} T^{2}},
$$

where $T$ is the symbol repetition interval and $f$ is the frequency. The parameter $\gamma^{2}$ can be expressed as

$$
\gamma^{2}=2 \pi B_{F W H M} T,
$$

where $B_{F W H M}$ is the full-width half-maximum bandwidth of the spectral line.

The information rate expressed in bits per channel symbol of a two-level construction with one pilot symbol per frame is

$$
R=\frac{R_{1} \cdot\left(M_{1}-1\right)+R_{2} \cdot M_{1} \cdot\left(M_{2}-1\right)}{M_{1} \cdot M_{2}},
$$

where $M_{1}-1$ is the number of symbols of code $\mathcal{C}_{1}$ in one frame, $R_{1}$ is the information rate of code $\mathcal{C}_{1}, M_{2}-1$ is the number of consecutive symbols of code $\mathcal{C}_{2}, R_{2}$ is the information rate of code $\mathcal{C}_{2}$, and $M_{1} \cdot M_{2}$ is the total number of symbols in one frame. In the example (1), illustrated in Fig. 1, one has $M_{1}=2, M_{2}=4$.

Iterative demodulation and decoding, as for instance described in [6], can be used after the first demodulation based on pilot symbols only. After having decoded the first-level code, the transmitted code word is regenerated and its symbols are used as pilot symbols in the second demodulation and decoding stage.

\section{Analysis of Channel CAPACity}

Let $\mathcal{X}_{p}$ be the deterministic sequence of pilot symbols and let $\mathcal{X}_{1}$ and $\mathcal{X}_{2}$ be the random processes of symbols of the firstlevel code and of the second-level code, respectively. Similarly, the received sequence is divided in three parts called $\mathcal{Y}_{p}, \mathcal{Y}_{1}$, $\mathcal{Y}_{2}$, where $\mathcal{Y}_{p}$ corresponds to the time instants where pilot symbols $\mathcal{X}_{p}$ are transmitted, while $\mathcal{Y}_{i}$ corresponds to the time instants where symbols of level $i$ are transmitted.

Let $x_{1}^{n}$ and $y_{1}^{n}$ denote the channel input vector $\left(x_{1}, x_{2}, \cdots, x_{n}\right)$ and the channel output vector $\left(y_{1}, y_{2}, \cdots, y_{n}\right)$, respectively. The information rate between $\mathcal{Y}$ and $\mathcal{X}$ is

$$
I(\mathcal{Y} ; \mathcal{X})=\lim _{n \rightarrow \infty} \frac{1}{n} I\left(y_{1}^{n} ; x_{1}^{n}\right) .
$$

By chain rule on $\mathcal{X}$ one writes

$$
I(\mathcal{Y} ; \mathcal{X})=I\left(\mathcal{Y} ; \mathcal{X}_{p}\right)+I\left(\mathcal{Y} ; \mathcal{X}_{1} \mid \mathcal{X}_{p}\right)+I\left(\mathcal{Y} ; \mathcal{X}_{2} \mid \mathcal{X}_{1}, \mathcal{X}_{p}\right),
$$

where, here and in what follows, the information rate of each one of the sub-channels is computed by dividing the information between vectors by the number of uses of the composite channel, e.g.

$$
I\left(\mathcal{Y} ; \mathcal{X}_{p}\right)=\lim _{n \rightarrow \infty} \frac{1}{n} I\left(y_{1}^{n} ; x_{p, 1}^{n}\right),
$$

where $x_{p, 1}^{n}$ is the vector of pilot symbols where zeros are inserted in the positions occupied by first-level and secondlevel coded symbols. Note that, since $\mathcal{X}_{p}$ is a known sequence,

$$
I\left(\mathcal{Y} ; \mathcal{X}_{p}\right)=0
$$

therefore

$$
I(\mathcal{Y} ; \mathcal{X})=I\left(\mathcal{Y} ; \mathcal{X}_{1} \mid \mathcal{X}_{p}\right)+I\left(\mathcal{Y} ; \mathcal{X}_{2} \mid \mathcal{X}_{1}, \mathcal{X}_{p}\right) .
$$

Invoking the chain rule on $\mathcal{Y}$, the first term in the right side of the above equation is

$$
I\left(\mathcal{Y} ; X_{1} \mid X_{p}\right)=I\left(\mathcal{Y}_{1}, \mathcal{Y}_{p} ; X_{1} \mid X_{p}\right)+I\left(\mathcal{Y}_{2} ; X_{1} \mid X_{p}, \mathcal{Y}_{1}, \mathcal{Y}_{p}\right)
$$

leading to

$$
\begin{aligned}
I(\mathcal{Y} ; X)= & I\left(\mathcal{Y}_{1}, \mathcal{Y}_{p} ; X_{1} \mid X_{p}\right)+I\left(\mathcal{Y} ; X_{2} \mid X_{1}, X_{p}\right) \\
& +I\left(\mathcal{Y}_{2} ; X_{1} \mid X_{p}, \mathcal{Y}_{1}, \mathcal{Y}_{p}\right) .
\end{aligned}
$$

The term $I\left(\mathcal{Y}_{2} ; \mathcal{X}_{1} \mid \mathcal{X}_{p}, \mathcal{Y}_{1}, \mathcal{Y}_{p}\right)$ appearing in the above equation is the contribution coming from the blind processing of $\mathcal{Y}_{2}$ at the first stage. In the numerical results presented in the next section we renounce to this contribution, which is small in many cases of practical interest. It can be computed from equation (4) as

$$
\begin{aligned}
I\left(\mathcal{Y}_{2} ; \mathcal{X}_{1} \mid \mathcal{X}_{p}, \mathcal{Y}_{1}, \mathcal{Y}_{p}\right)= & I(\mathcal{Y} ; \mathcal{X})-I\left(\mathcal{Y}_{1}, \mathcal{Y}_{p} ; \mathcal{X}_{1} \mid \mathcal{X}_{p}\right) \\
& -I\left(\mathcal{Y} ; \mathcal{X}_{2} \mid \mathcal{X}_{1}, \mathcal{X}_{p}\right)
\end{aligned}
$$




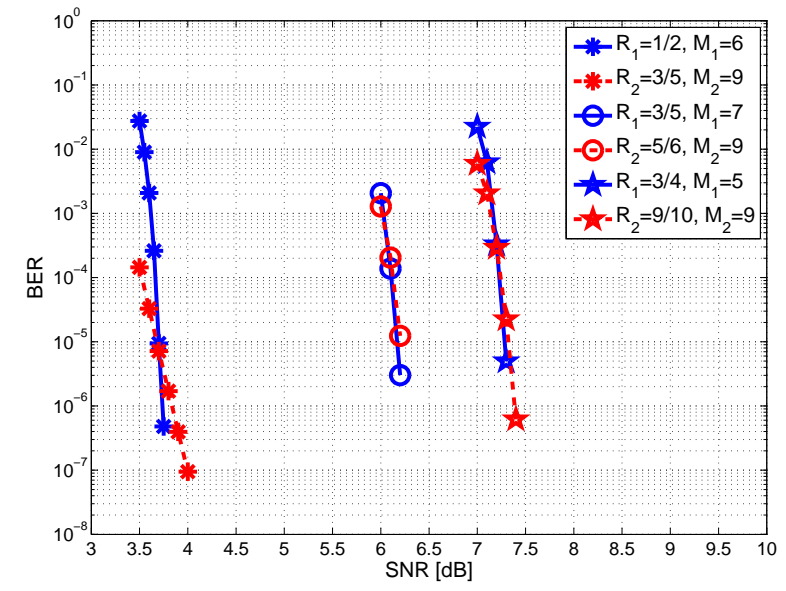

Fig. 2. 4-QAM, $\gamma=0.125$, LDPC codes of length 64800 from the DVBS2 standard. Performance of individual codes with iterative demodulation and decoding [6]. Solid line: first-level code. Dashed line: second-level code. The second-level code assumes ideal decoding of the first-level code.

where the three terms in the right side of (5) can be computed as in [12]. Specifically, for $I(\mathcal{Y} ; \mathcal{X})$ one uses pilot symbols inserted with period $M_{1} \cdot M_{2}$, for $I\left(\mathcal{Y} ; \mathcal{X}_{2} \mid \mathcal{X}_{1}, \mathcal{X}_{p}\right)$ one uses pilot symbols inserted with period $M_{2}$, while for $I\left(\mathcal{Y}_{1}, \mathcal{Y}_{p} ; \mathcal{X}_{1} \mid \mathcal{X}_{p}\right)$ one uses pilot symbols inserted with period $M_{1}$ and Wiener phase noise with step

$$
\gamma \sqrt{M_{2}}
$$

\section{NUMERICAL RESUlts}

Numerical results have been derived using low-density parity-check (LDPC) codes as component codes. Designing the matrix of the component codes is out of the scope of the present paper. To demonstrate the benefits of the proposed scheme we adopt the LDPC family of the popular digital video broadcasting - satellite (DVB-S2) standard, whose matrices are of public domain, thus making our results easy to reproduce. Fig. 2 reports the performance of the component codes of three two-level schemes, the component codes being decoded according to [6]. The performance of the second-level code is obtained by assuming no errors from the first-level code. This a realistic assumption for capacity-achieving codes, as LDPC codes are provided that they are operating in the waterfall region. For capacity-achieving systems the performance of the two-level scheme is dominated by the performance of the worst of the two component codes, hence, in a good design, the bit error rate (BER) curves of the two components codes should be close to each other, as it happens with the codes of Fig. 2. In Fig. 3 and 4 our proposed scheme is compared to the classical one-level scheme for 4-ary quadrature amplitude modulation (QAM) and 16-QAM, respectively. The number of iterations of the LDPC decoder of the one-level scheme is the same as the average number of iterations of the two LDPC decoders of our scheme. It should be observed that, in the proposed scheme, it turns out to be convenient to make more iterations at the first level and less iterations at the second

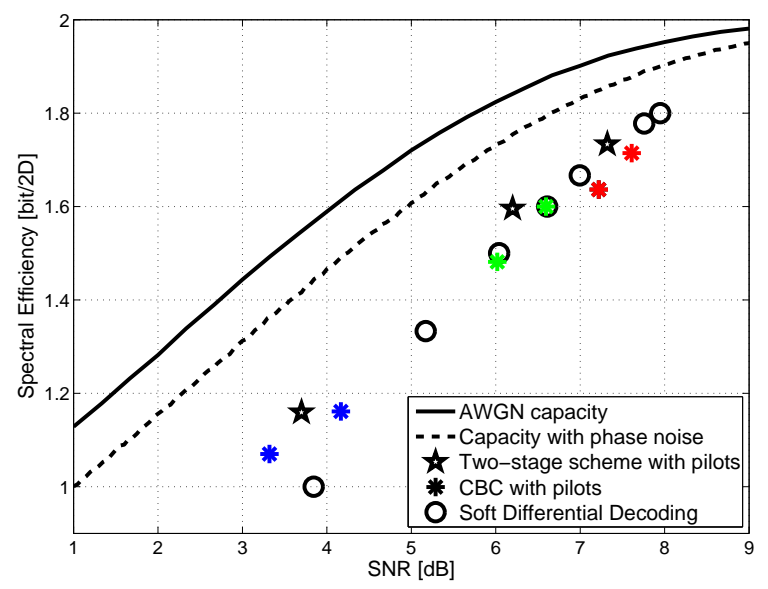

Fig. 3. 4-QAM, $\gamma=0.125$. The two-stage scheme is based on one pilot symbol per frame and the three two-level codes of Fig. 2. CBC indicates one-level coding with the algorithm of [6], while soft differential decoding indicates the algorithm of [13]. The performance is evaluated at bit error rate of $10^{-5}$ after 24 iterations.

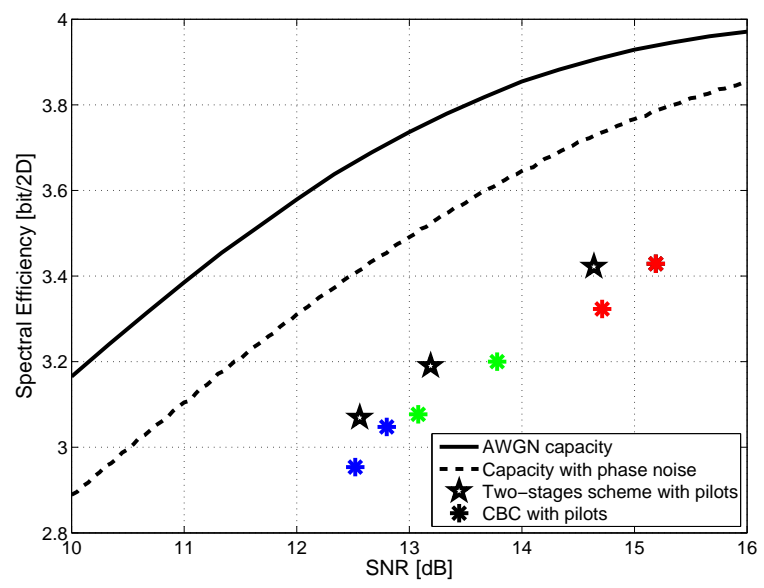

Fig. 4. 16-QAM, $\gamma=0.125$. The two-stage scheme is based on one pilot symbol per frame and $M_{1}=7, M_{2}=9$. CBC indicates one-level coding with the algorithm of [6]. The performance is evaluated at bit error rate of $10^{-5}$ after 24 iterations.

level. In Figs. 3 the performance of [13] is also reported, even if it should be said that [13], where demodulation is based on a trellis, is much more demanding in terms of complexity compared to the adversaries. Moreover, [13] is based on differential demodulation and it is suited only for phase shift keying (PSK)-type constellations therefore it cannot be applied to 16-QAM. From Figs. 3 and 4 it appears the advantage of our scheme, especially with 16-QAM, where it brings system performance closer to the capacity curve of about $0.5 \mathrm{~dB}$ compared to the adversary.

\section{CONCLUSions}

In the paper, a staged demodulation and decoding method is proposed for channels affected by strong phase noise. The 


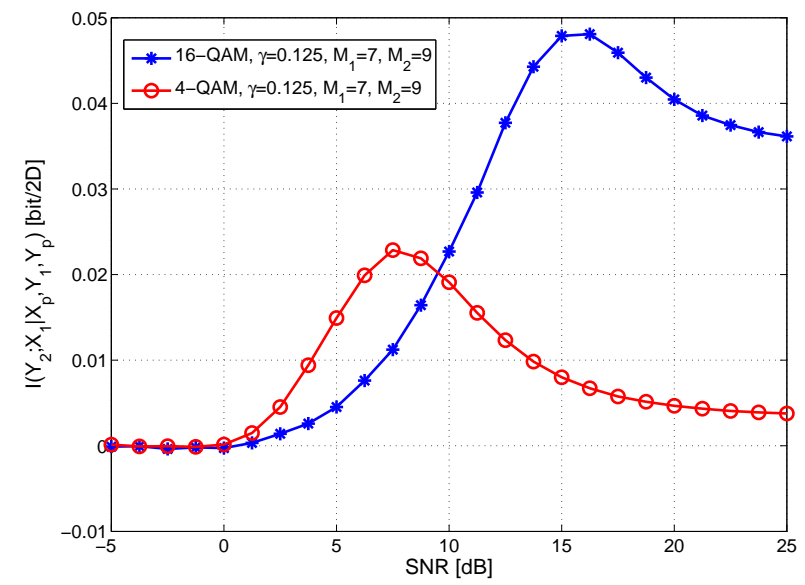

Fig. 5. 4-QAM and 16-QAM, $M_{1}=7, M_{2}=9, \gamma=0.125$. The figure shows the term $I\left(\mathcal{Y}_{2} ; \mathcal{X}_{1} \mid \mathcal{X}_{p}, \mathcal{Y}_{1}, \mathcal{Y}_{p}\right)$ of equation (5).

results presented in the paper show that the proposed method outperforms adversary methods base on conventional onelevel demodulation and decoding. We should mention that some margin still exists to improve the method, as it can be seen from the results on channel capacity reported in Fig. 5. Specifically, Fig. 5 shows the capacity that one loses when renounces to help the first-level demodulator by blind processing the constellation symbols of the second-level code. The 0.05 bits/2D of capacity loss with 16 QAM at SNR $=15 \mathrm{~dB}$ can be converted in decibels by the popular law of $3 \mathrm{~dB} / \mathrm{bit}$, leading to a potential margin of $0.15 \mathrm{~dB}$ coming from the mentioned blind processing. Before concluding the paper, it is worth adding that, although here only results for Wiener phase noise have been presented, the staged demodulation and decoding scheme can be adopted also to combat phase noise of higher order, for instance the one proposed in [18], which can better fit oscillators used in radio systems.

\section{REFERENCES}

[1] G. J. Foschini, G. Vannucci, "Characterizing filtered light waves corrupted by phase noise," IEEE Trans. Inform. Theory, vol. 34, no. 6, pp. 1437-1448, Nov. 1988.

[2] M. Magarini, A. Spalvieri, F. Vacondio, M. Bertolini, M. Pepe, and G. Gavioli, "Empirical modeling and simulation of phase noise in longhaul coherent optical systems," Optics Express, vol. 19, issue 23, pp. 22455-22461, Nov. 7, 2011.

[3] M. G. Taylor, "Phase estimation methods for optical coherent detection using digital signal processing," IEEE J. Lightw. Technol., vol. 27, no. 7, pp. 901-914, April 1, 2009.

[4] T. Pfau, S. Hoffmann, R. Noe, "Hardware-efficient coherent digital receiver concept with feedforward carrier recovery for M-QAM constellations," IEEE J. Lightw. Technol., vol. 27, no. 8, pp. 989-999, April 15, 2009.

[5] X. Li, Y. Cao, S. Yu, W. Gu, and Y. Ji "A simplified feedforward carrier recovery algorithm for coherent optical QAM systems," IEEE J. Lightw. Technol., vol. 29, no. 5, pp. 801-807, March 1, 2011.

[6] G. Colavolpe, A. Barbieri, and G. Caire, "Algorithms for iterative decoding in the presence of strong phase noise," IEEE Journal on Selected Areas in Communications, vol. 23, n. 9, pp. 1748-1757, Sept. 2005.
[7] A. Barbieri and G. Colavolpe, "Soft-output decoding of rotationally invariant codes over channels with phase noise," IEEE Trans. on Commun., vol. 55, n. 11, pp. 2125-2133, Nov. 2007.

[8] A. Barbieri and G. Colavolpe, "On the information rate and repeataccumulate code design for phase noise channel", IEEE Trans. on Commun., vol. 59, n. 12, pp. 3223-3228, Dec. 2011.

[9] L. Barletta, M. Magarini, and A. Spalvieri, "Estimate of information rates of discrete-time first-order Markov phase noise channel", IEEE Photon Technol. Lett., vol 23, no. 21, pp. 1582-1584, Nov.1, 2011.

[10] A. Spalvieri and L. Barletta, "Pilot-aided carrier recovery in the presence of phase noise", IEEE Trans. Commun., vol. 59, no. 7, pp. 1966-1974, July 2011.

[11] M. Magarini, L. Barletta, A. Spalvieri, F. Vacondio, T. Pfau, M. Pepe, M. Bertolini, and G. Gavioli, "Pilot-symbols-aided carrier-phase recovery for 100-G PM-QPSK digital coherent receivers," IEEE Photonics Technol. Letters, vol. 24, issue 9, pp. 739-741, May 01, 2012.

[12] L. Barletta, M. Magarini, and A. Spalvieri, "The information rate transferred through the discrete-time Wiener's phase noise channel", IEEE J. Lightw. Technol., vol. 30, no. 10, pp. 1480-1486, May 15, 2012.

[13] M. Peleg, S. Shamai (Shitz), and S. Galan, "Iterative decoding for coded noncoherent MPSK communications over phase-noisy AWGN channel," Proc. IEE Commun., vol. 147, pp. 87-95, Apr. 2000.

[14] M. V. Eyuboglu "Detection of coded modulation signals on linear severely distorted channels using decision-feedback noise prediction and interleaving", IEEE Trans. Commun., vol. 36, no. 4, pp. 401-409, April 1988.

[15] H. D. Pfister, J. B. Soriaga, and P. H. Siegel, "On the achievable information rates for finite state ISI channels," Proc. IEEE Global telecommun. Conf., S. Antonio, Texas, Nov. 2001, pp. 2992-2996.

[16] T. Li and O. M. Collins, "A successive decoding strategy for channels with memory", IEEE Trans. Inf. Theory, vol. 53, no. 2, pp. 628-646, February 2007.

[17] S. Das and P. Schniter, "Noncoherent communication over the doubly selective channel via successive decoding and channel re-estimation", in Proc. annu. Allerton Conf. Communications, Control and Computing, Monticello, IL, 2007.

[18] A. Spalvieri and M. Magarini, "Wiener's analysis of the discrete-time phase-locked loop with loop delay," IEEE Trans. Circuits and Systems II, vol. 55, no. 6, pp. 596-600, June 2008. 